\title{
CHARACTERISTICS OF INTERNATIONAL CLINICAL PRACTICE GUIDELINES FOR THE PHARMACOLOGICAL TREATMENT OF DEPRESSION
}

\author{
F. Gabriel ${ }^{1,4}$, C. G. R. C. Molino ${ }^{1,4}$, N. C. L. Santos ${ }^{1,4}$, R. O. Valentim ${ }^{1,4}$, D. O. de Melo ${ }^{2,4}$, E. Ribeiro ${ }^{1,3,4}$. \\ ${ }^{1}$ Faculdade de Ciências Farmacêuticas da Universidade de São Paulo, São Paulo, Brasil. \\ 2 Instituto de Ciências Ambientais, Químicas e Farmacêuticas da Universidade Federal de São Paulo, São Paulo, Brasil. \\ ${ }^{3}$ Hospital Universitário da Universidade de São Paulo, São Paulo, Brasil. \\ ${ }^{4}$ Chronic Diseases and Informed Decisions (Chronide) Group.
}

\section{OBJECTIVES:}

To characterize international Clinical Practice Guidelines (CPGs) for the pharmacological treatment of depression.

\section{BACKGROUND:}

Depression is a severe health issue that affects 300 million people worldwide.

CPGs are a valuable tool for guiding patient care and improving the treatment of patients with depression.

\section{MATERIALS AND METHODS:}

We systematically searched documents containing recommendations for the pharmacological treatment of adults with depression in primary care using Medline, Embase, Cochrane, and 12 specific CPGs databases (DBs). Only articles published in English, Portuguese, or Spanish between January 2011 and August 2017 were included. Data on CPGs characteristics were extracted by two reviewers (Figure 1). Any discrepancies were resolved by consensus (as seen in Molino et al., 2019).

\section{RESULTS AND CONCLUSIONS:}

A total of 28 CPGs were selected. Of these, most $(64 \%, 18 / 28)$ originated from developed countries, mainly the United States and Canada, and half were developed by government institutions. Further, less than half $(43 \%, 12 / 28)$ were developed using a systematic literature review or formal adaptation process, and only 36\% (10/28) used the Grading of Recommendations Assessment, Development and Evaluation or its adaptation. Patient representatives were included in three $(11 \% ; 3 / 28)$ CPGs development teams.

Few CPGs contain recommendations based on a systematic review of the literature, indicating that CPGs development generally does not involve the use of tools to evaluate scientific evidence. Such tools should be used to allow health professionals to assess the strength of recommendations for clinical practice. Moreover, including patient representatives in CPGs development teams should be more widely considered as this is fundamental in improving the applicability of CPGs.



Copyright @ 2019 Franciele Cordeiro Gabriel - francordegabriel@gmail.com

References:

AGREE Next Steps Consortium (2017). The AGREE II Instrument [Electronic version]. Retrieved from http://www.agreetrust.org. Molino CGRC et al. (2019). JAMA Intern Med. doi: 10.1001/jamainternmed.2018.7529 